\title{
Strain propagation in artificial extracellular matrix proteins can accelerate cell spreading and polarization $\dagger$
}

\begin{abstract}
Shelly Tzlilt*a and David A. Tirrell ${ }^{\text {ab }}$
In recent years the ability of cells to sense the mechanical properties of their environments and deform them locally has become increasingly clear. To better understand the mechanical coupling between cells and their surroundings, we have examined the dynamics of adhesion of Chinese hamster ovary cells cultured on engineered protein substrates with different viscoelastic properties. We find that cell spreading and polarization rates vary two- and five-fold, respectively, for crosslinked and uncrosslinked proteins, despite the fact that the rates of growth of individual adhesion complexes on the different substrates are comparable. A wave of adhesion growth along the cell contour is observed by total internal reflection fluorescence microscopy for cells plated on crosslinked materials, but not on uncrosslinked substrates. We propose a mechanism in which cell-induced strains accumulate in crosslinked materials as a result of adhesion growth. Strain propagation within the material explains the observed adhesion growth patterns and the increased rates of spreading and polarization characteristic of cells cultured on crosslinked substrates. We investigate the proposed mechanism through Brownian dynamics simulation.
\end{abstract}

Received 15th September 2012 Accepted 29th April 2013

DOI: $10.1039 / \mathrm{c} 3 \mathrm{sm} 27137 d$

www.rsc.org/softmatter

\section{Results and discussion}

\section{Cell spreading and polarization}

CHO-K1 cells were allowed to spread on spin-coated films of artificial extracellular matrix (aECM) proteins that were crosslinked to varying extents. The aECM substrates combine domains derived from fibronectin and elastin, and carry lysine residues within the elastin domains to allow crosslinking by difunctional electrophiles (Fig. S1 $\dagger$ ). We have shown previously that such aECM proteins can be crosslinked to yield materials with elastic moduli similar to those of natural elastins ${ }^{\mathbf{1 0 , 1 1}}$ and that incorporation of the tenth Type III domain of fibronectin promotes cell spreading and adhesion. ${ }^{12}$ Here we used bis(sulfosuccinimidyl suberate) (BS3) as the crosslinker, and we identify aECM substrates by the ratio of equivalents of lysine residues in the protein to activated ester groups in the crosslinker (e.g., aECM1:2).

Fig. 1 shows the rates of cell spreading and polarization on highly crosslinked (aECM1:2) and uncrosslinked (aECM1:0) substrates (see Fig. $\mathrm{S} 2 \uparrow$ for the rates on aECM substrates with intermediate degrees of crosslinking). The rates of cell spreading and polarization were measured separately for individual cells and then averaged. For each cell, the projected area and aspect ratio (ratio of the major to minor axis of the best-fit ellipse) were plotted as functions of time and the slopes of the linear parts of the curves were defined as the spreading and polarization rates, respectively (see Materials and methods for details). The spreading and polarization rates increased 2.2-fold and 5-fold, respectively, as the degree of crosslinking increased. 

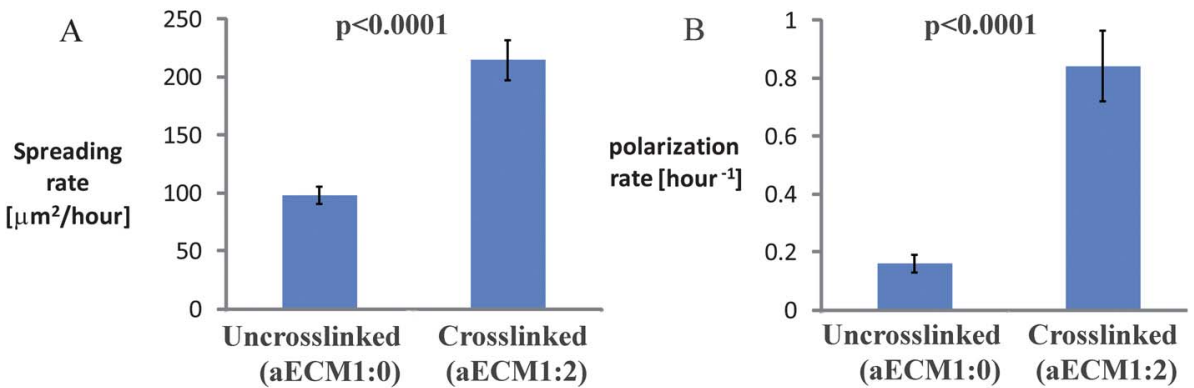

$\mathrm{C}$
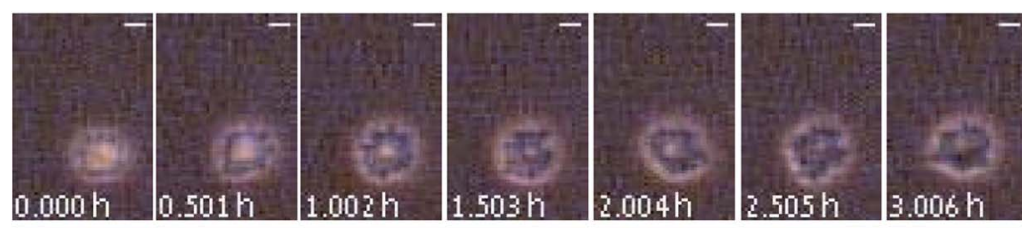

$\mathrm{D}$

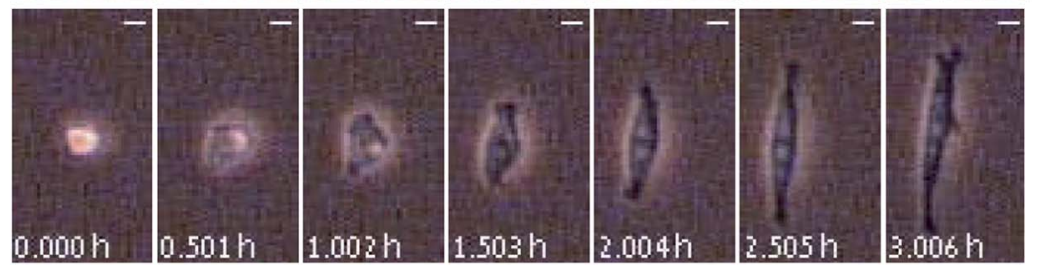

Fig. 1 The rates of CHO-K1 cell spreading (A) and polarization (B) on uncrosslinked (aECM1:0) and highly crosslinked (aECM1:2) substrates. Error bars represent s.e.m. Time sequence images of single cell spreading and polarization are shown for CHO-K1 cells on aECM1:2 (C) and aECM1:0 (D). The scale bar corresponds to 10 $\mu \mathrm{m}$. At least 40 cells were used for the analysis of each substrate in at least three independent experiments. $p$-values were determined according to the unpaired one-tailed Mann-Whitney test.

Increased rates of cell spreading on stiff, crosslinked substrates have been reported previously, and have been correlated with the formation of well organized actin stress fibers anchored at their ends to the ECM through mature focal adhesions. ${ }^{13-15}$ It is well established that cell adhesion requires clustering of adhesion receptors and their ECM ligands into adhesion complexes., ${ }^{4,16,17}$ Often, ligand clustering is accomplished by substrate remodeling (Fig. S3†). Consequently, one might expect the spreading process to be faster on materials that are permissive with respect to remodeling. The results shown in Fig. 1 do not follow this logic. Crosslinking constrains ligand movement and matrix remodeling; however, it correlates with an increase in the rate of cell spreading.

\section{Adhesion dynamics}

In order to explore further the relations between substrate mechanics and cellular dynamics, CHO-K1 cells were transiently transfected with eGFP fused to paxillin, a protein known to associate closely with adhesions. ${ }^{18,19}$ Adhesion dynamics were monitored by live cell total internal reflection fluorescence microscopy (TIRFM). The rates of growth of individual adhesions (see Materials and methods) were comparable on both highly crosslinked (aECM1:2) and uncrosslinked (aECM1:0) substrates $\left(0.30 \pm 0.03 \mu \mathrm{m}^{2} \mathrm{~min}^{-1}\right.$ vs. $0.31 \pm 0.03 \mu \mathrm{m}^{2} \min ^{-1}$ $(n=100)$, respectively) with no statistically significant difference. However, on the crosslinked substrates, adhesion growth followed a striking pattern. Adhesions grew in waves along the periphery of the cell; the position of maximal adhesion growth rate traveled along the edge of the cell at a speed of $2.7 \pm 0.2 \mu \mathrm{m}$ $\min ^{-1}(n=10)$ (Fig. 2 and Videos S1 and S2 $\uparrow$ ). We saw no evidence of spatial correlation in the growth of adhesions on uncrosslinked substrates. Correlated growth of adhesion complexes can explain how the rate of cell spreading can vary with the degree of crosslinking when the adhesion growth rate does not, since spreading is a consequence of collective behavior of cell-substrate adhesions.

Different types of waves have been described in the literature. ${ }^{20-23}$ The adhesion waves observed in this work are reminiscent of membrane ruffling, in which cycles of protrusion and retraction of lamellipodia propagate along the periphery of the cell. ${ }^{21-23}$ Because membrane ruffling has been correlated with travelling waves of Rho GTPase activity, it has been suggested to be a consequence of transmission of polymerization forces through the actin network. ${ }^{21}$ Whether or not the adhesion waves observed in this work are related to membrane ruffling, our results show that they are critically dependent on the mechanical properties of the substrate.

\section{Brownian dynamics simulation}

Crosslinking of artificial extracellular matrix proteins changes the viscoelastic properties of the substrate. Crosslinked substrates behave as viscoelastic solids that are able to store mechanical deformations (i.e., $G^{\prime} / G^{\prime \prime}>1$ where $G^{\prime}$ is the elastic (storage) modulus and $G^{\prime \prime}$ is the viscous (loss) modulus). In contrast, uncrosslinked substrates behave as viscoelastic liquids $\left(G^{\prime} / G^{\prime \prime}<1\right)$, which dissipate most of the energy 
A
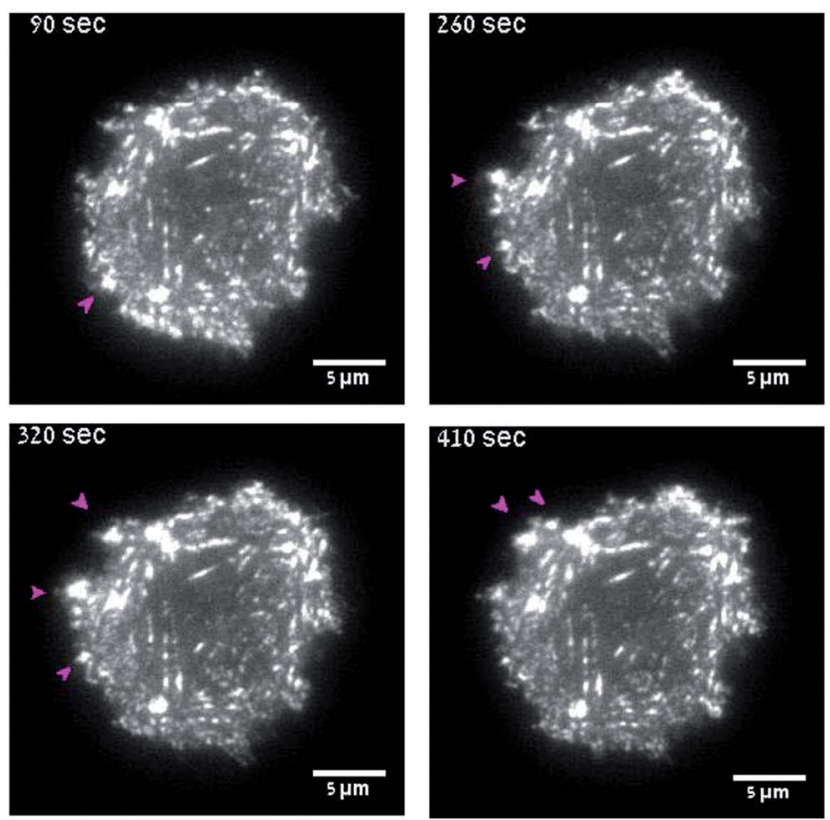

B

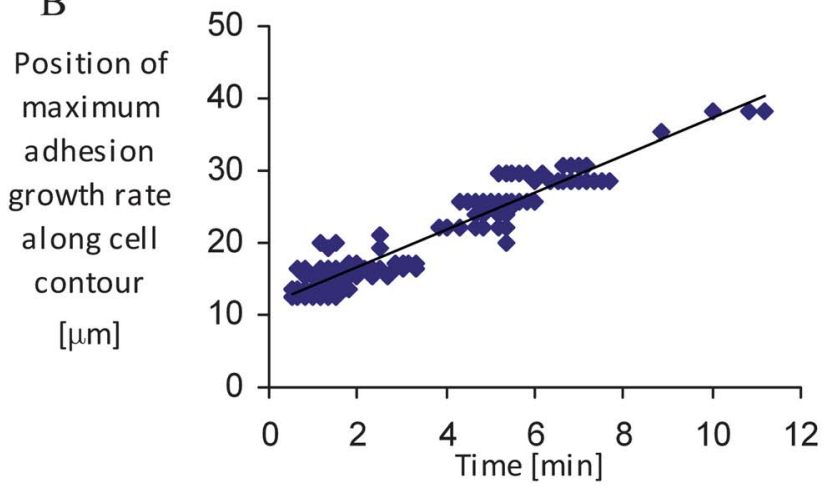

Fig. 2 (A) Dynamics of paxillin-labeled adhesions during cell spreading on aECM1:2. The pink arrows indicate positions of maximal growth rate. (B) Position of maximal growth rate along cell contour as a function of time obtained for the cell shown in A. $p$-values according to the Spearman correlation test were less than or equal to 0.001 for all cells.

associated with mechanical deformation, (see Fig. $\mathrm{S} 4 \dagger$ for microrheology measurements of the two substrates). It should be noted however, that the elastic (storage) modulus, $G^{\prime}$, and the viscous (loss) modulus, $G^{\prime \prime}$ are frequency dependent properties. The relevant frequency window is that in which cells act to deform the matrix, which is not known for CHO cells. For the purposes of comparing matrices, we focused on the range of frequencies $(0.1-10 \mathrm{~Hz})$ characteristic of the beating of cardiac cells ${ }^{24,25}$ and membrane ruffling. ${ }^{26}$ In this regime $G^{\prime} / G^{\prime \prime}$ for the highly crosslinked material is significantly larger than 1 (Fig. $\mathrm{S} 4 \dagger$ ).

To examine the role of energy storage by the matrix in modulating the formation of adhesion complexes, we turned to computer simulation. A schematic illustration of the model is shown in Fig. 3. The substrate, for the case of a viscoelastic solid (the limit of $G^{\prime} / G^{\prime \prime}>1$ ), is modeled as a hexagonal lattice of

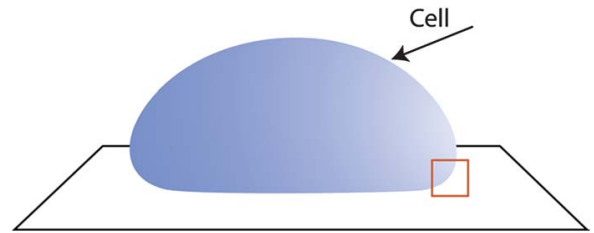

A

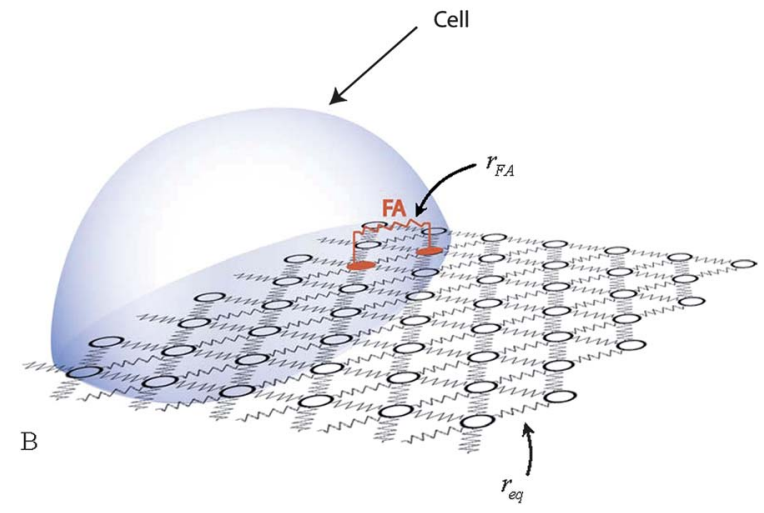

Fig. 3 Schematic illustration of the simulation model. (B) is a magnified view of the cell edge (the red square in $(A)$ ). The aECM substrate is modeled as a hexagonal lattice of beads. Nearest neighbors are connected by harmonic springs. Beads that are part of an adhesion complex are colored red. $r_{\text {eq }}$ and $r_{\text {FA }}$ denote the spring equilibrium length and the average distance between beads in an adhesion complex, respectively. An adhesion grows by increasing the number of substrate ligands (beads) in the complex. Since $r_{\mathrm{FA}}<r_{\text {eq, }}$ adhesion growth deforms the substrate.

beads, in which nearest-neighbor beads are connected by harmonic springs. The beads and springs represent the viscous and elastic components of the matrix, respectively. In this coarse-grained model, the beads represent the matrix-bound ligands that mediate cell adhesion. Adhesion growth requires addition of new ligands (beads) to engage cell receptors, and is driven by short-range attraction between cell receptors (e.g., integrins). The equilibrium distance between beads in the matrix (the equilibrium length of the springs) is larger than the average distance between beads in an adhesion complex (which corresponds to the minimum in the receptor-receptor interaction potential). Consequently, the growth of adhesion complexes, which is defined by aggregation of ligands, is associated with deformation of the lattice.

The energy required to aggregate ligands and deform the lattice is provided by the cell. The cell exerts force pulses of defined duration and frequency on randomly chosen adhesion complexes (see Materials and methods and ESI $\dagger$ for details). Essentially, only the material is modeled in the simulation. Ligand aggregation is interpreted as adhesion growth and the presence of the cell is felt through the force pulses generated by the cell and through the initial distribution of adhesion nuclei (taken to be uniform along the cell contour; see Materials and methods for additional details).

The pattern of adhesion growth observed experimentally is reproduced in the simulation. Sequential growth of adhesion complexes occurs in the simulation along the path of maximum 
compressive deformation in the material (Fig. 4, S5 and Videos $\mathrm{S} 3$ and $\mathrm{S} 4 \dagger$ ). Compression reduces the distance between beads and thereby reduces the barrier for ligand aggregation and subsequently for adhesion growth. The tendency of adhesions to grow around an 'adhesion seed' results both in correlated adhesion growth and in the formation of adhesions in clusters, which in turn leads to polarized distributions of adhesions. Both the correlated growth of adhesions, and their non-uniform distribution disappear for very soft matrices, for which the barrier for matrix deformation is low (see Fig. 5 and Video S5 $\dagger$ ). Moreover, the polarized adhesion distribution is coupled to cell shape. On soft matrices, the cell-substrate contact surface remains circular throughout the simulation, while on stiff matrices, cells develop polarized shapes (see Fig. S9†). The emergence of cell polarization as a function of matrix rigidity was modeled previously by assuming positive feedback between cell contractile forces and local matrix rigidity. ${ }^{27}$ In our simulation, there is no need for an explicit positive feedback loop. The forces applied by the cell are spatially uniform and neither their amplitude nor their frequency depends on adhesion size. Therefore, the correlated adhesion growth in the simulation can be mediated only by the material.

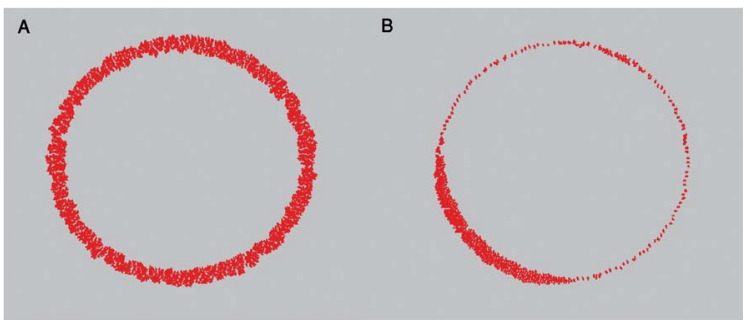

Fig. 5 Adhesion configuration for soft $\left((\mathrm{A}) ; K=200 k_{\mathrm{B}} T / r_{\mathrm{eq}}{ }^{2}\right)$ and rigid $((\mathrm{B}) ; K=$ $\left.1000 k_{\mathrm{B}} T / r_{\mathrm{eq}}{ }^{2}\right)$ matrices. For highly crosslinked matrices (B), an adhesion growth event (which is associated with deformation in the matrix) acts as a seed for an adhesion cluster as a result of the reduction in the barrier for adhesion formation in a pre-deformed matrix region. For lightly crosslinked matrices (A), the barrier for adhesion formation is lower and consequently this effect is not significant. Adhesion formation is uncorrelated (see also Video $55 \dagger$ ).

If the dimensions of the simulation box are on the order of a few cell diameters, the cells in the simulation acquire polarized configurations characterized by two large, diametrically opposed adhesion clusters (Fig. S6 $\dagger$ ). This polarized arrangement of cell adhesion complexes is a result of coupling between opposite edges of the cell, and is mediated by matrix deformation.
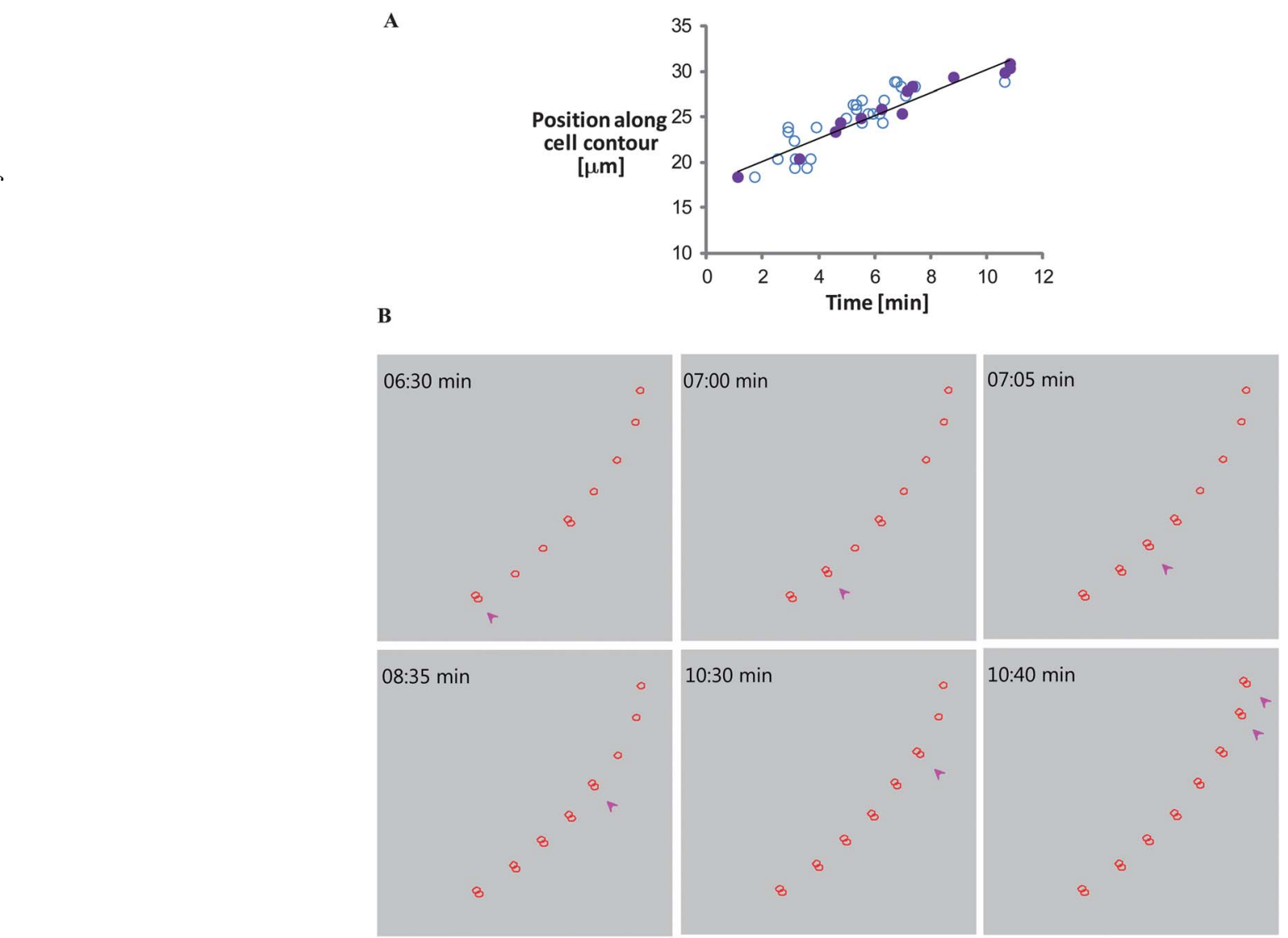

Fig. 4 Dynamics of cell adhesion growth as obtained from Brownian dynamics simulation with $r_{\mathrm{FA}}=0.6 r_{\mathrm{eq}}$ and $d=500 \mathrm{~nm}$ (for additional parameter values and details, see ESI†). (A) Position of maximal growth rate (filled circles) and maximal compressive deformation (empty circles) along the cell contour as a function of time. (B) The corresponding snapshots from the simulation. Only a small part of the cell contour is shown. Only beads that are part of an adhesion complex are displayed. The pink arrows indicate the position of maximal adhesion growth rate. Notice that adhesions grow sequentially along the cell contour. See Video $53 \dagger$ for full bead configuration. 
Deformation caused by adhesion formation at the "top" edge of the cell, deforms the material on the "bottom" edge of the cell as a consequence of the periodic boundary conditions. The size of the simulation box defines the effective cell density; a small simulation box corresponds to high cell density and allows cells to communicate through substrate deformation to synchronize their polarization states. The ability of cells to communicate through mechanical deformation of soft solid substrates was recently demonstrated for fibroblast alignment, collective cell migration and synchronized beating of embryonic cardiomyocytes. ${ }^{28-31}$

\section{Concluding remarks}

The rates of spreading and polarization of $\mathrm{CHO}-\mathrm{K} 1$ cells cultured on engineered protein substrates increase with the crosslink density and elasticity of the substrate. Brownian dynamics simulations show that cell-induced deformation of the substrate can lead to correlated growth of adhesion complexes and enhanced cell spreading, behavior that is observed experimentally by TIRF microscopy. The simulation reveals a major difference in the way adhesions develop on soft versus rigid substrates. While on soft matrices adhesions form in an isotropic manner (see Video S5 $\dagger$ ), on rigid matrices adhesions form non-isotropically around adhesion seeds (see Videos S4 and S6†). In the simulation, deformation of the substrate is a consequence of ligand clustering during adhesion growth. Adhesion growth results in both tensile and compressive deformation. Compressive deformation reduces the barrier for ligand aggregation and promotes growth of adhesion complexes; extension has the opposite effect. Consequently, the deformation field generated in the material can determine not only the position and growth rate of adhesions, but also their orientation and shape. The ratio of storage and loss moduli $\left(G^{\prime} / G^{\prime \prime}\right)$ provides a measure of the extent to which a material stores and propagates mechanical deformation. Consequently, phenomena mediated by propagation of mechanical deformation in the substrate should correlate with $G^{\prime} / G^{\prime \prime}$ and the extent of crosslinking. In contrast, the rate of growth of single adhesion complexes is not strongly dependent on the degree of crosslinking since two opposite effects counterbalance one another. On one hand, cellular forces acting at one position on highly crosslinked materials can lead to material deformation and consequently to adhesion formation in neighboring positions. On the other hand, a high degree of crosslinking results in higher elastic modulus and consequently higher deformation energy. The simulation further suggests that synchronized cell polarization can be induced by cell-cell interaction mediated by material deformation.

\section{Materials and methods}

\section{Cell culture}

CHO-K1 cells were cultured in Dulbecco's modified Eagle's medium (DMEM), supplemented with 10\% fetal bovine serum, $1 \%$ non-essential amino acids, $1 \%$ sodium pyruvate and $1 \%$ penicillin-streptomycin (growth media-GM). Cell transfection was conducted using Lipofectamine 2000 (Invitrogen). Cells from passages 2 to 10 were used.

\section{Preparation of spin-coated aECM films}

Round glass coverslips ( $25 \mathrm{~mm}$ diameter; no. 0) were sonicated in a mixture of ethanol and $\mathrm{KOH}$ for $15 \mathrm{~min}$ and rinsed several times with distilled $\mathrm{H}_{2} \mathrm{O}$. aECM protein solutions were prepared by dissolving the protein $\left(100 \mathrm{mg} \mathrm{mL}^{-1}\right.$ in $\left.\mathrm{ddH}_{2} \mathrm{O}\right)$ for $4 \mathrm{~h}$ at $4{ }^{\circ} \mathrm{C}$. Bis(sulfosuccinimidyl) suberate (BS3) was used to crosslink the aECM protein substrates. Protein-BS3 solutions with stoichiometric ratios of lysine amines in the aECM protein to active esters in BS3 of $1: 0,1: 0.1,1: 0.4,1: 1$ and $1: 2$ were prepared. BS3-protein solutions $(100 \mu \mathrm{L})$ were spin-coated at $3000 \mathrm{rpm}$ for $45 \mathrm{~s}$. Each protein film was stored overnight at $4{ }^{\circ} \mathrm{C}$ before use. The thickness of the hydrated films was determined by atomic force microscopy to be in the range of $1-2.5 \mu \mathrm{m}$ for all substrates. Variations in thickness were observed for the highly crosslinked material as well as for the uncrosslinked one. The observed spreading behavior, however, was very consistent with no significant variation between samples with the same degree of crosslinking.

\section{Cell spreading assay}

aECM-coated coverslips were mounted in a 6-well tissue culture plate by using sterile clear silicone glue (GE Silicone RTV108). CHO-K1 cells $\left(2 \times 10^{5}\right)$ were incubated at $37^{\circ} \mathrm{C}$ for $10 \mathrm{~min}$ with $0.5 \mathrm{mg}$ of GRGDSP peptide (Calbiochem) in $1 \mathrm{~mL}$ serum free medium (SFM). The cell suspension was added to a well containing SFM and the plate was incubated for $40 \mathrm{~min}$ at $37^{\circ} \mathrm{C}$ to allow the inhibited cells to attach non-specifically to the substrate. Next, the medium was changed to normal growth medium (GM) with no peptide and the plate was put on the microscope. A chamber was set up around the microscope to maintain the microscope stage and chamber interior at $37{ }^{\circ} \mathrm{C}$ with $5 \% \mathrm{CO}_{2}-95 \%$ air to sustain cells. A hole was opened in the cover of one of the empty wells in the 6-well plate and an air supply was attached to ensure that cells were maintained under the $\mathrm{CO}_{2}$-air mixture. Water was also added to surrounding empty wells to prevent excessive evaporation of the medium. Cell morphology was followed for $12 \mathrm{~h}$ by time-lapse phase contrast microscopy on a Nikon Eclipse TE300 microscope at $10 \times$ magnification. Digital images of 3-5 different fields of view for each substrate were acquired every $10 \mathrm{~min}$ using MetaMorph v6.3.2 (Molecular Devices, Sunnyvale, CA). Cell morphology was traced manually using ImageJ v1.37 (NIH, USA, http:// rsb.info.nih.gov/ij/). Cell projected area and aspect ratio were measured for each cell as a function of time. Cell aspect ratio was calculated by fitting the cell projected area to an ellipse and calculating the ratio of major and minor axes. The spreading (change in cell projected area) and polarization (change in cell aspect ratio) curves for single cells could be divided into a lag phase, spreading/polarization phase and occasionally a plateau phase. The spreading/polarization phases could be fitted to a linear curve. The rates of spreading and polarization were taken as the slopes of these lines (Fig. S7 $\dagger$ ). The reported rates were obtained by averaging the rates calculated from videos recorded in at least three independent experiments. At least 40 cells were used for the analysis of each substrate. 


\section{TIRFM}

TIRFM experiments were done under conditions identical to those used for the cell spreading assay. To allow for high resolution microscopy, a $14 \mathrm{~mm}$ diameter opening was cut in the center of a standard $35 \mathrm{~mm}$ Corning culture dish and the aECMcoated coverslip was attached to the bottom of the dish covering the opening. The dish was maintained at $37{ }^{\circ} \mathrm{C}$ with $5 \%$ $\mathrm{CO}_{2}-95 \%$ air using a stage-mounted culture dish incubator (Warner Instruments). TIRF images were obtained using an Olympus IX71 inverted microscope (1.45 NA (oil) PlanApo $\times 100$ ) equipped with a TIRF illuminator. eGFP was excited with a $488 \mathrm{~nm}$ argon laser (Melles Griot). Images were taken for 30 min in $10 \mathrm{~s}$ intervals at $100 \times$ magnification using an iXON EMCCD camera (Andor). Custom-written MATLAB code was used to identify and monitor the formation and growth of paxillin/eGFP-labeled adhesions. Adhesion growth rate was defined as the slope of the linear fit to the plot of adhesion size as a function of time (Fig. $\mathbf{5 8} \dagger$ ). Ten cells were used for analysis of each substrate. 100 adhesions were used to calculate single adhesion growth rate for each substrate.

\section{Brownian dynamics simulation}

We used Brownian dynamics simulation ${ }^{32}$ to study the coupling between ligand clustering and material deformation in viscoelastic substrates. The material was modeled as a hexagonal lattice of $250 \times 250$ beads. Nearest-neighbor beads are connected by springs of equilibrium length $r_{\text {eq }}$. Hydrodynamic interactions between beads are neglected. In every time step of the simulation, the positions of all beads that are not part of an adhesion are updated according to:

$$
\boldsymbol{r}(t+\Delta t)=\boldsymbol{r}(t)+\boldsymbol{F}(t) \times \Delta t / \xi+\boldsymbol{r}_{\mathrm{G}}
$$

where $\boldsymbol{F}(t)$ is the sum of the forces the bead experiences as a result of its interactions with neighboring beads (through the springs) and of the forces applied by the cell, $\xi$ is the friction constant associated with a bead, and $\boldsymbol{r}_{\mathrm{G}}$ is a random displacement arising from random collisions with solvent molecules. $\boldsymbol{r}_{\mathrm{G}}$ is generated from a Gaussian distribution with zero mean and a variance of $\left\langle\boldsymbol{r}_{\mathrm{G}}{ }^{2}\right\rangle=\Delta t \times 2 k_{\mathrm{B}} T / \xi$.

The time step in the simulation $\Delta t$ is chosen such that the energy associated with a bead displacement $\Delta r=\left\langle\boldsymbol{r}_{\mathrm{G}}{ }^{2}\right\rangle^{1 / 2}$ is on the order of $k_{\mathrm{B}} T$ and $\Delta t \ll \tau_{\text {network }}$ where $\tau_{\text {network }}$ is the characteristic relaxation time of the material.

At the initial time point of the simulation, adhesion nuclei are distributed uniformly along the cell periphery with spacing $d$. In every step of the simulation, there is a probability $w_{\mathrm{F}} \times \Delta t$ that a force pulse will be applied to any given adhesion (where $w_{\mathrm{F}}$ is the frequency of force pulses in the cell). The force pulls (toward the adhesion complex) on a randomly chosen bead at the interface of the adhesion. If the distance between two beads, $r_{i j}$ - one of which is part of an adhesion (say $i$ ) - becomes smaller than $1.1 r_{\mathrm{FA}}$ (i.e., $r_{i j} \leq 1.1 r_{\mathrm{FA}}$ ), bead $j$ becomes part of the adhesion complex.

Notice that there is no explicit positive feedback loop in the simulation. The forces applied by the cell are spatially uniform and neither their amplitudes nor their frequencies depend on adhesion size. Very different forms of cell-generated forces give the same qualitative results (e.g., continuous sinusoidal force that acts on all adhesions either with the same phase or with random phases). For simplicity, adhesion growth in the simulation is irreversible.

All simulations were done in the limit of fast network relaxation time compared to the cell-generated-force frequency. In all simulations, the values of the energy supplied by the cell and the energy required to deform the network were comparable.

The simulation results were not sensitive to the parameters of choice. For a list of parameter values used to generate Fig. 4, 5, S5 and S6, see ESI. $\dagger$

Material deformations in the simulation are generated either by transient cell-generated forces or by adhesion growth (i.e., bead aggregation). To detect only material deformations that occur as a direct result of adhesion growth, the deformations reported in Fig. 4 and $\mathrm{S} 5 \dagger$ are calculated for every time point by equilibrating the bead position for the given adhesion configuration (obtained from the full simulation at time $t$ ) with no cell-generated forces.

\section{Acknowledgements}

We acknowledge Dr Eileen Fong for discussion and help in protein expression. We also thank Dr Zhen-Gang Wang for advice on the simulation model. S.T. was supported by the Human Frontier Science Program Cross-Disciplinary Fellowship. This work was supported by the NSF Center for the Science and Engineering of Materials and by NSF DMR 1206121 at the California Institute of Technology.

\section{References}

1 A. J. Engler, S. Sen, H. L. Sweeney and D. E. Discher, Cell, 2006, 126, 677-689.

2 A. D. Bershadsky, N. Q. Balaban and B. Geiger, Annu. Rev. Cell Dev. Biol., 2003, 19, 677-695.

3 F. Chowdhury, S. Na, D. Li, Y. C. Poh, T. S. Tanaka, F. Wang and N. Wang, Nat. Mater., 2010, 9, 82-88.

4 B. Geiger, J. P. Spatz and A. D. Bershadsky, Nat. Rev. Mol. Cell Biol., 2009, 10, 21-33.

5 C. M. Lo, H. B. Wang, M. Dembo and Y. L. Wang, Biophys. J., 2000, 79, 144-152.

6 J. Y. Wong, A. Velasco, P. Rajagopalan and Q. Pham, Langmuir, 2003, 19, 1908-1913.

7 A. E. X. Brown and D. E. Discher, Curr. Biol., 2009, 19, R781R789.

8 K. Franze, J. Gerdelmann, M. Weick, T. Betz, S. Pawlizak, M. Lakadamyali, J. Bayer, K. Rillich, M. Gogler, Y. B. Lu, A. Reichenbach, P. Janmey and J. Kas, Biophys. J., 2009, 97, 1883-1890.

9 A. del Rio, R. Perez-Jimenez, R. C. Liu, P. Roca-Cusachs, J. M. Fernandez and M. P. Sheetz, Science, 2009, 323, 638641.

10 K. Di Zio and D. A. Tirrell, Macromolecules, 2003, 36, 15531558. 
11 P. J. Nowatzki and D. A. Tirrell, Biomaterials, 2004, 25, 12611267.

12 E. Fong and D. A. Tirrell, Adv. Mater., 2010, 22, 5271-5275.

13 N. Q. Balaban, U. S. Schwarz, D. Riveline, P. Goichberg, G. Tzur, I. Sabanay, D. Mahalu, S. Safran, A. Bershadsky, L. Addadi and B. Geiger, Nat. Cell Biol., 2001, 3, 466-472.

14 P. C. Georges and P. A. Janmey, J. Appl. Physiol., 2005, 98, 1547-1553.

15 R. J. Pelham and Y. L. Wang, Proc. Natl. Acad. Sci. U. S. A., 1997, 94, 13661-13665.

16 G. Maheshwari, G. Brown, D. A. Lauffenburger, A. Wells and L. G. Griffith, J. Cell Sci., 2000, 113, 1677-1686.

17 H. J. Kong, T. R. Polte, E. Alsberg and D. J. Mooney, Proc. Natl. Acad. Sci. U. S. A., 2005, 102, 4300-4305.

18 E. Zamir, B. Z. Katz, S. Aota, K. M. Yamada, B. Geiger and Z. Kam, J. Cell Sci., 1999, 112, 1655-1669.

19 C. K. Choi, M. Vicente-Manzanares, J. Zareno, L. A. Whitmore, A. Mogilner and A. R. Horwitz, Nat. Cell Biol., 2008, 10, 1039-1050.

20 L. B. Case and C. M. Waterman, PLoS One, 2011, 6, e26631.

21 M. Machacek and G. Danuser, Biophys. J., 2006, 90, 14391452.

22 M. Machacek, L. Hodgson, C. Welch, H. Elliott, O. Pertz, P. Nalbant, A. Abell, G. L. Johnson, K. M. Hahn and G. Danuser, Nature, 2009, 461, 99-103.
23 H. G. Dobereiner, B. J. Dubin-Thaler, J. M. Hofman, H. S. Xenias, T. N. Sims, G. Giannone, M. L. Dustin, C. H. Wiggins and M. P. Sheetz, Phys. Rev. Lett., 2006, 97, 038102.

24 A. J. Engler, C. Carag-Krieger, C. P. Johnson, M. Raab, H. Y. Tang, D. W. Speicher, J. W. Sanger, J. M. Sanger and D. E. Discher, J. Cell Sci., 2008, 121, 3794-3802.

25 A. A. Werdich, E. A. Lima, I. Dzhura, M. V. Singh, J. Li, M. E. Anderson and F. J. Baudenbacher, Am. J. Physiol.: Heart Circ. Physiol., 2008, 294, H2352-H2362.

26 G. Giannone, B. J. Dubin-Thaler, H. G. Dobereiner, N. Kieffer, A. R. Bresnick and M. P. Sheetz, Cell, 2004, 116, 431-443.

27 A. Zemel, F. Rehfeldt, A. E. X. Brown, D. E. Discher and S. A. Safran, Nat. Phys., 2010, 6, 468-473.

28 C. A. Reinhart-King, M. Dembo and D. A. Hammer, Biophys. J., 2008, 95, 6044-6051.

29 J. P. Winer, S. Oake and P. A. Janmey, PLoS One, 2009, 4, e6382.

30 T. E. Angelini, E. Hannezo, X. Trepat, J. J. Fredberg and D. A. Weitz, Phys. Rev. Lett., 2010, 104, 168104.

31 X. Tang, P. Bajaj, R. Bashir and T. A. Saif, Soft Matter, 2011, 7, 6151-6158.

32 D. L. Ermak and J. A. McCammon, J. Chem. Phys., 1978, 69, 1352-1360. 